\title{
Minimal invasive Chirurgie - eine Zwischenbilanz
}

Die minimal invasive Chirurge wird nicht ohne Grund als die dritte patientenfreundliche Revolution in der Chirurgie nach Einführung der Asepsis und der Anästhesie bezeichnet. Trotz der bekannten Vorteile, die inzwischen in zahlreichen Studien, zum Teil auch auf höchster Stufe in der Hierarchie der evidenzbasierten Medizin, bestätigt werden konnten, ist bisher nur die laparoskopische Cholezystektomie zum flächendeckenden Standard geworden; aber selbst hier liegt die Durchführungsrate bei nur 70-80\%, wobei zudem noch mit einer Konversionsrate von annähernd $10 \%$ zu rechnen ist. Die Gründe hierfür sind vielfältig: Zum einen ist es die vollkommen neue Technik - videoendoskopisch, ein vollständig neues Instrumentarium, das zweidimensionale Bild mit völlig neuer Sehgewohnheit, der Verzicht auf die Hand zur Organexposition, zur Gewebeschichten- und zur Strukturenidentifikation sowie zum raschen Eingreifen bei Komplikationen - und zum anderen werden die Kosten der laparoskopischen Operation, die dem Krankenhaus entstehen, höher eingeschätzt als für einen entsprechenden konventionellen Eingriff.

Als ein Meilenstein in der Weiterentwicklung dieser neuen revolutionären Technik ist die diesjährige gemeinsame Jahrestagung der drei deutschsprachigen Arbeitsgemeinschaften Chirurgische Arbeitsgemeinschaft für Minimal Invasive Chirurgie (CAMIC) der Deutschen Gesellschaft für Viszeralchirurgie, Arbeitsgemeinschaft für Minimal Invasive Chirurgie (AMIC), Österreich, sowie die Schweizerische Arbeitsgemeinschaft für Laparo- und Tho- rakoskopische Chirurgie (SALTC) einzustufen, die vom 19. bis 21. Februar 2004 in Salzburg unter Leitung von Herrn Professor Hans Werner Waclawiczek nun bereits zum dritten Mal stattfand. Hauptanliegen der Tagung war es, nach 15 Jahren minimal invasiver Chirurgie eine Zwischenbilanz zu ziehen. Im Mittelpunkt der Diskussionen standen die Fragen, was bereits Standard ist, was Routine, aber noch kein Standard ist und schließlich wie die zukünftige Entwicklung sein wird. Höhepunkte der Tagung waren die «State of the art»-Referate zu einigen der wichtigsten Themen in der minimal invasiven Chirurgie, vorgetragen von international anerkannten Experten. Erfreulicherweise ist es uns gelungen, die Autoren zu gewinnen, ihre Vorträge als Übersichtsarbeiten in einem Supplement der Chirurgische Gastroenterologie InterDISZIPLINÄR zu veröffentlichen. Uns ist bewusst, dass dies für die Autoren mit nicht unerheblicher zusätzlicher Arbeit verbunden war. Allen Autoren sei daher ganz herzlich gedankt. Wir sind überzeugt, dass ihre Beiträge auch eine wesentliche Hilfe sind für die Entscheidungsprozesse der täglichen Routine.

Erlauben Sie uns, Sie bereits jetzt zur 4. gemeinsamen Tagung der drei Arbeitsgemeinschaften einzuladen. Sie wird vom 28. bis 30. April 2005 in Berlin unter der Leitung von Dr. Eckhard Bärlehner stattfinden.

R. Bittner, Stuttgart H.W. Waclawiczek, Salzburg

$\begin{array}{ll}\text { KARGER } & \text { @ 2004 S. Karger GmbH, Freiburg } \\ \begin{array}{l}\text { Fax +497614520714 } \\ \begin{array}{l}\text { E-mail Information@Karger.de } \\ \text { www.karger.com }\end{array}\end{array} & \begin{array}{l}\text { Accessible online at: } \\ \text { www.karger.com/cga }\end{array}\end{array}$

www.karger.com
Prof. Dr. Dr. h.c. Reinhard Bittner

Klinik für Allgemein- und Visceralchirurgie

Marienhospital Stuttgart

Böheimstraße 37, D-70199 Stuttgart

E-mail reinhardbittner@vinzenz.de 